\title{
Neural Network Technology applied in the Diagnosis of Ovarian Tumors Lihong Bao
}

Medical College, Inner Mongolia University for the Nationalities, Tongliao, Inner Mongolia, 028000, China

baolihong71@163.com

Keywords: Feature extraction, artifical neural network, Cell recognition

\begin{abstract}
The original ovarian cells from ascites are preprocessed and samples are gained in the paper, .Features parameters of morphology are extracted from images of cells samples.The images of cells samples are recognized and classified by Multilayer Perceptron Neural Network and Radial Basis Function Neural Network.Several arithmetics of MLPNN and RBFNN are discussed,and cross entropy arithmetic are suggested.Among the recognized results, the recognition rate and classification of RBFNN and MLPNN with BP arithmetic based on adaptive are the best one.
\end{abstract}

\section{Introduction}

At present, digital images processing, pattern recognition and artificial intelligence are widely applied in the fields of biology and medicine.Some achievements have been gained by these techniques.But there is few reports on the pathologic diagnosis system which can be used to recognize cells images in the mean of neural network.In the paper,based in the detailed investigation of ovarian cells from ascites images recognition and feature extraction,results of utilizing neural network to diagnose and classify cells images are satisfiable and valuable in clinical application.

\section{Materials and Methods}

All objects (patients before operation) should take fasting venous $3 \mathrm{ml} 4 \mathrm{~h}$ centrifugation serum specimens in the early morning, excluding hemolytic samples, so as to be stored in the refrigerator with the temperature of $-80^{\circ} \mathrm{C}$. The method of detecting serum HE4 can use ELISA method, the kit case is provided by Swedish FU-JIREBIO Company, while the diagnosis instrument is provided by BIO-RAD America, which is Coda automatic enzyme immunoassay analyzer; the method of detecting serum CA125 can use chemiluminescence method, besides the instrument is Abbott ARCHITECT i2000SR, American full automatic chemiluminescence immunoassay analyzer, whose reagent is the original set of product. Moreover, the process of detection is in strict accordance with the operating instructions of instrument and reagent, at the same time, carrying on two kinds of quality control detection, namely, the low value and high value detection, so as to ensure the accuracy of the experimental results. The normal reference range of serum HE4 and CA125 is 0pmol/L-70.0 pmol/L and 0kU/L-35.0kU/L respectively.

\section{Outline of PNN}

Probabilistic neural network (PNN) is a common network model, which is based on Bayesian classifier and probabilistic function [6]. PNN has a wide range of applications in model identification, time series prediction, signal processing, as well as fault diagnosis and other fields [7-10]. The PNN is a pattern classifier that combines the widely used Bayes decision strategy with the Parzen nonparametric estimator for estimation of probability density functions of different classes. Unlike other neural network architectures, PNN is easy to implement and the network is easily interpretable [11].

Generally, the probabilistic density function is the normal probabilistic density function as follows. 


$$
f_{A}(X)=\frac{1}{(2 \pi)^{\frac{m}{2}} \sigma^{m}}\left(\frac{1}{n_{A}}\right) \sum_{p=1}^{n_{A}} \exp \left(-\frac{\left(X-X_{A p}\right)^{\prime}\left(X-X_{A p}\right)}{2 \sigma^{2}}\right)
$$

where $f_{A}(X)$ represents the value of probabilistic density function of Category A at point X; $m$ represents the number of input variables; $\sigma$ represents smooth parameter; $n_{A}$ represents the number of training vectors in Category A; $X$ represents the testing data vectors; $X_{A p}$ represents the p-th training data in Category A.

Because

$$
\begin{gathered}
\frac{1}{(2 \pi)^{\frac{m}{2}} \sigma^{m}}\left(\frac{1}{n_{A}}\right)=\text { constant }=h \\
\left(\mathbf{X}-\mathbf{X}_{A p}\right)^{\prime}\left(\mathbf{X}-\mathbf{X}_{A p}\right)=\sum_{i=1}^{m}\left(x_{i}-x_{i}^{A p}\right)^{2}
\end{gathered}
$$

the probabilistic density function can be simplified as follows

where

$$
f_{A}(X)=h \sum_{p=1}^{n_{A}} f_{A p}
$$

$$
f_{A p}=\exp \left(-\frac{\sum_{i=1}^{m}\left(x_{i}-x_{i}^{A p}\right)^{2}}{2 \sigma^{2}}\right)
$$

where $x_{i}$ represents the value of $\mathrm{i}$-th input variable in the testing sample; $x_{i}^{A p}$ represents the i-th input variable of the p-th sample of Category A in the sample base.

The PNN network is simply a parallel 4-layer structure: input, pattern, summation, and decision layers (Fig. 1). The input layer receives and normalizes input vector; each unit in pattern layer represents a training vector with response function $\exp \left[\left(\mathbf{X}^{\prime} \mathbf{X}_{A p}-1\right) / \sigma^{2}\right]$. Summation layer computes the summation of each pattern and multiplies the loss factor. Decision layer selects the largest one in summation layer as the classification result.

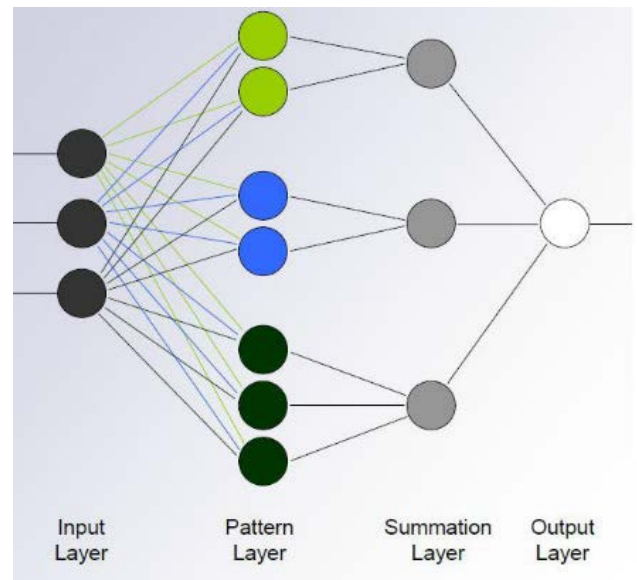

Fig. 1 The structure of PNN

\section{Statistical Analysis Method}

Statistical analysis can be carried out by using SPSS11.5 software. Measurement data can use $\overline{\mathrm{x}}$ $\pm \mathrm{s}$ to represent, when $\mathrm{P}<0.05$, it can represent the difference is statistically significant. 


\section{The Detection Results of Serum HE4 and CA125 in the Health Control Group, Ovarian Benign Disease Group and Ovarian Cancer Group}

The level of serum HE4 and CA125 in ovarian cancer group is significantly higher than that in healthy control group and ovarian benign disease group. Besides, the differences are statistically significant ( $\mathrm{P}<0.1$ ); The level of serum CA125 in ovarian benign disease group serum is higher than that in healthy control group, and the difference is also statistically significant $(\mathrm{P}<0.5)$, while the level of serum HE4 in ovarian benign disease group compared with that in normal control group, there is no statistically significant difference $(\mathrm{P}>0105)$, which can be shown in Table 1 :

\begin{tabular}{cccc} 
Table 1 & \multicolumn{4}{c}{ Comparison of Serum HE4 and CA125 Level $(\overline{\mathrm{x}} \pm \mathrm{s})$ among } \\
\hline $\begin{array}{c}\text { Number of Group } \\
\text { The Healthy Control } \\
\begin{array}{c}\text { Group } \\
\text { Ovarian Benign } \\
\text { Disease Group } \\
\text { Ovarian Cancer } \\
\text { Group }\end{array}\end{array}$ & 66 & $58.36 \pm 12.12$ & CA125(kU/L \\
\hline
\end{tabular}

Note: compared with the health control group, $\mathrm{P}>0.05 ; * *$ compared with the health control group, $\mathrm{P}<0.05 ; * * *$ compared with the health control group and ovarian benign disease group, $\mathrm{P}<0.01$

\section{The Evaluation of Single and Combined Detection of Serum HE4 and CA125 on Diagnostic Effect of Ovarian Cancer}

Among the twelve cases of patients with early ovarian cancer, the sensitivity of the combined detection of HE4+CA125 was 50\%, the sensitivity of the detection of serum HE4 was $41.7 \%$, while CA125 was only 25\%; among the thirty five cases of ovarian cancer, the sensitivity of combined detection of serum HE4 and CA125 were higher than that of the single detection, but the specificity was decreased compared with HE4 single detection , which can be shown in Table 2:

Table 2 The Evaluation of Serum HE4 and CA125 on Diagnostic Effect of Ovarian Cancer

\begin{tabular}{cccccc}
\hline $\begin{array}{c}\text { Tumor } \\
\text { markers }\end{array}$ & $\begin{array}{c}\text { Sensitivity } \\
(\%)\end{array}$ & $\begin{array}{c}\text { Specificity } \\
(\%)\end{array}$ & $\begin{array}{c}\text { Accuracy } \\
(\%)\end{array}$ & $\begin{array}{c}\text { Positive } \\
\text { predictive } \\
\text { value } \\
(\%)\end{array}$ & $\begin{array}{c}\text { Negative } \\
\text { predictive } \\
\text { value }(\%)\end{array}$ \\
\hline HE4 & $74.3(26 / 35)$ & $91.3(115 / 126)$ & $87.6(141 / 161)$ & $70.3(26 / 37)$ & $92.7(115 / 124)$ \\
CA125 & $80.0(28 / 35)$ & $65.1(82 / 126)$ & $68.3(110 / 161)$ & $38.9(28 / 72)$ & $92.1(82 / 89)$ \\
HE4+CA125 & $82.9(29 / 35)$ & $81.7(103 / 126)$ & $82.0(132 / 161)$ & $55.8(29 / 52)$ & $94.5(103 / 109)$ \\
\hline
\end{tabular}

\section{Discussion}

The incidence of ovarian cancer is occult, whose inspection is lack of specificity and sensitivity, which is also lack of diagnosis method, when it is found out, it usually is in late stage, therefore, it has become a health problem in the world. In recent years, due to the number of the infertile women and the increase of environmental pollution and other factors, the incidence of ovarian cancer showed a gradual upward trend in China. If we can improve the cure rate of the ovarian cancer will undoubtedly, if we can find out in the early diagnosis, CA125 as a tumor marker has been widely used in clinical diagnosis of ovarian cancer. However, because of the ovarian benign disease and malignant disease, endometriosis, pelvic inflammation, peritonitis and chest liver and kidney disease, and so on, which can cause the elevation of serum CA125, so the application of CA125 diagnosis ovarian cancer is not accurate, sometimes it can lead to unnecessary operation. Therefore, it needs more sensitive and specific serum tumor markers in the clinical diagnosis. 
During the period of diagnosis on ovarian cancer, the detection on sensitivity of CA125 is similar to the detection of HE4, but the specificity and accuracy of the former is significantly higher; the combined detection on sensitivity of HE4+CA125 is the highest (82.9\%), but the specificity, accuracy and positive predictive value is slightly lower than that of the detection of HE4, which may be affected by the high limitation of false positive rate of CA125. In the early stage of ovarian cancer, the sensitivity of combined detection of HE4+CA125 is 50\%, HE4 is 41.7\%, while CA125 is only $25 \%$. Therefore, HE4 is a better tumor marker for the early diagnosis of ovarian cancer.

\section{Conclusion}

In the paper, the linear relationship between the classified effect are studied. Features, classification and recognition rate increase. But if there are linear relationship between the features , redundant data reduce recognition rate. Combined computer technology with practical experiences of pathology experts and based on processing techniques of medicine images, neural network can be used to recognize cells images and shows its significant value in clinical diagnosis and medical research at present and in the future.

\section{Acknowledgments}

This paper was supported by the 2015 Inner Mongolia Higher Science Project: "Nerve growth factor and its receptor in ovarian tissue” (NJZY171)

\section{Reference}

[1] P K Sahoo, S Soltani, A K C Wong,and Y C Chen. Survery of thresholding techniques[J]. Computer Vision, Graphics, and Image Processing,1988, 41(2):233-260

[2] Baldi P and K Hornik. Neural Networks and Principal Component Analysis:Learning from Examples without Local Minimum[J].Neural Networks,1989, 1:53-58.

[3] Hornik K.Some New Results on Neural Network Approxination [J].Neural Networks, 1993, 6:1069-1072.

[4] Moody J,Darken C. Fast learning in networks of locally-tuned processing units[J]. Neural Networks, 1989, 1:281-294

[5]SimonHaykin. Neuralnetworks: A comprehensive foundation,2nd edition[M].Beijing:China Machine Press,2004, 209-210 